\title{
\begin{tabular}{l|l} 
Mibraries & DSpace@MIT
\end{tabular}
}

\author{
MIT Open Access Articles
}

Connecting electronic entropy to empirically accessible electronic properties in high temperature systems

The MIT Faculty has made this article openly available. Please share how this access benefits you. Your story matters.

Citation: Rinzler, Charles C., and Antoine Allanore. “Connecting Electronic Entropy to Empirically Accessible Electronic Properties in High Temperature Systems." Philosophical Magazine 96, 29 (September 2016): 3041-3053 (C) 2016 Informa UK limited, trading as Taylor \& Francis group

As Published: https://doi.org/10.1080/14786435.2016.1216657

Publisher: Taylor \& Francis

Persistent URL: http://hdl.handle.net/1721.1/114780

Version: Author's final manuscript: final author's manuscript post peer review, without publisher's formatting or copy editing

Terms of use: Creative Commons Attribution-Noncommercial-Share Alike 


\section{Connecting Electronic Entropy to Empirically Accessible Electronic Properties in High Temperature Systems}

Charles C. Rinzler ${ }^{2}$ and Antoine Allanore ${ }^{1}$

${ }_{1}^{1}$ Massachusetts Institute of Technology, Department of Materials Science and Engineering 77 Massachusetts Avenue, Room 13-5066, Cambridge, MA, USA 02139

e-mail address: allanore@mit.edu

$617-452-2758$

${ }^{2}$ Massachusetts Institute of Technology, Department of Materials Science and Engineering 77 Massachusetts Avenue, Room 13-5095, Cambridge, MA, USA 02139

e-mail address: rinzler@mit.edu

617-314-1999 


\title{
Connecting Electronic Entropy to Empirically Accessible Electronic Properties in High Temperature Systems
}

\author{
A quantitative theoretical model connecting the thermopower and electronic entropy of \\ molten systems is proposed, the validity of which is tested for semiconductors and metallic \\ materials. The model accurately provides the entropy of mixing for molten semiconductors, \\ as shown for the representative system Te-Tl. Predictions of the electronic entropy of \\ fusion for compounds are in agreement with available data and offer a novel means to \\ identify the correct electrical conductivity model when Hall measurements are not \\ available. Electronic entropy for molten semiconductor and metallic systems is shown to \\ reflect order in the molten and solid state. The model proves accurate at predicting the \\ electronic state entropy contribution to the electronic entropy of mixing.
}

Keywords: entropy; electronic entropy; thermopower; molten semiconductor

PACS: $64,71,81$

\section{Introduction}

Electronic entropy is a manifestation of the accessible state-space of electrons and consequently reflects the structure of condensed matter systems. Empirical access to electronic entropy therefore offers a means of probing the electronic ordering of matter. To date, in materials thermodynamics, the contribution of electronic entropy to the total entropy or, more importantly, to the entropy variation across phases, is often ignored or neglected [1]. As claimed in Fultz [2], the study of electronic entropy remains a frontier for high-temperature thermodynamics. A quantitative connection of electronic entropy to an experimentally accessible quantity would then allow rapid investigation and improved prediction of ordering in high temperature systems. We therefore herein propose a model connecting the thermopower and electronic entropy of systems 
at high temperature. The model is first used to successfully predict the thermopower and entropy of mixing of the molten Te-Tl system with accuracy comparable to other empirical methods. The robustness of the model is then tested for the electronic entropies of fusion of selected compounds and for the entropy of mixing of a molten metallic system. The proposed approach proves to offer novel access to ordering and mixing properties in molten metallic and semiconductor systems. We show that in certain cases, here at high temperatures, electronic entropy contributes significantly to the total entropy of a system.

\section{Electronic Entropy and Thermopower}

We propose to measure the total electronic entropy of high temperature systems through the measurement of macroscopic electronic properties. The microscopic basis for the connection between thermopower and entropy has previously been discussed by Peterson and Chaikin [3,4], and the statistical mechanical basis for the electronic entropy has been presented in Wallace [5]. Prior work by Rockwood has proposed the following connection between the partial molar electronic entropy of a system $\left(\overline{S_{e}}\right.$, a macroscopic thermodynamic property of the system, in J mole $^{-1} \mathrm{~K}^{-1}$ ) and the thermopower [6-8]:

$$
\overline{S_{e}}=-F \alpha,
$$

where we understand $\overline{S_{e}}$ as defined as:

$$
\overline{S_{e}}=\left(\frac{d S}{d n_{e}}\right)_{T, P, N}
$$


where $S$ is the total entropy $\left(\mathrm{J} \mathrm{mol}^{-1} \mathrm{~K}^{-1}\right), n_{e}$ refers to the number of moles of mobile electrons per mole of system, $F$ is Faraday's Constant, and the thermopower $\left(\alpha, \mathrm{VK}^{-1}\right)$ is classically defined in terms of a measured difference in electrical potential $(\phi)$ as a function of temperature [9]:

$$
\frac{\Delta \phi}{\Delta T}=-\alpha .
$$

The reader is invited to consider Rockwood, Kjelstrup, and Tykodi for a historical perspective on the connection between thermopower and electronic entropy $[8,10-12]$. It should be noted that there has been much discussion in the literature, starting with Thompson, about the possibility of a quantitative connection between irreversible thermodynamic properties, such as the thermopower or Peltier coefficient, and reversible thermodynamic properties, such as the entropy or heat capacity.

We seek to provide empirical evidence that quantitatively connects thermopower to electronic entropy. To leverage available experimental entropy data, it is useful to work in the context of integral instead of partial molar entropy. From Eq. (2) we can define:

$$
S_{e}^{p}=n_{e} \overline{S_{e}} .
$$

Leveraging Eq. (1) we can reframe Eq. (4) in terms of the thermopower:

$$
S_{e}^{p}=-n_{e} F \alpha,
$$

in units of $\mathrm{J} \mathrm{mol}^{-1} \mathrm{~K}^{-1}$ or, equivalently:

$$
S_{e}^{p}=-n e \alpha,
$$


in units of $\mathrm{J} \mathrm{m}^{-3} \mathrm{~K}^{-1}$ where $n$ is density of electrons $\left(\mathrm{m}^{-3}\right)$ that contribute to nonlocal transport phenomena and $e$ is the fundamental charge. For materials where the conducting particle has positive effective charge (e.g. holes) Eq. (6) reads:

$$
S_{e}^{p}=n e \alpha,
$$

where $n$ is now the density of positive charge carriers that contribute to nonlocal transport phenomenai.

Thus, it is in principle possible to macroscopically probe the electronic properties of a system (thermopower and number of carriers) and relate them to an essential thermodynamic quantity: entropy. As can be demonstrated starting from thermodynamics of solutions (see Appendix), electronic entropy is the critical driving factor for the thermodynamic behavior of certain systems, including molten semiconductors. In such systems, evaluating the electronic entropy of mixing, $\Delta S_{e}$, provides an approximation of the total entropy of mixing $\Delta S_{\text {mix }}$.

\section{Application to Molten Semiconductors}

To verify the proposed connection both electronic and thermodynamic data of high temperature systems are required. There is a dearth of available electronic data at high temperatures. However, one class of material system, molten semiconductors, has been studied in detail precisely because of the comprising members' unique electronic properties. Consequently, we will leverage the available datasets for these systems to evaluate the predicting performances of the proposed model.

Molten semiconductors exhibit electronic properties characteristic of solid semiconductors in their liquid phase (the molten state). Herein the term semiconductor refers to systems that 
have an optical band gap and an increase in electronic conductivity with temperature. Sulfides, oxides, selenides, tellurides, and other systems of commercial relevance exhibit these features [13]. A strong body of work describes the physical basis of the electronic behavior of molten semiconductors [14] and indicates that for many systems the Drude model holds for electronic conductivity [15].

Applying the Drude model of conductivity to mobile electrons allows connecting the density of mobile electrons, $n$, to two empirically accessible quantities, the conductivity $\left(\sigma, \mathrm{ohm}^{-1}\right.$ $\left.\mathrm{m}^{-1}\right)$ and mobility $\left(\mu, \mathrm{m}^{2} \mathrm{~V}^{-1} \mathrm{~s}^{-1}\right)$ :

$$
\sigma=n e \mu .
$$

For materials for which the Drude model holds, the connection between $S_{e}^{p}$ and the electronic properties therefore follows for negatively charged carriers:

$$
S_{e}^{p}=-\frac{\sigma}{\mu} \alpha
$$

and for positively charged carriers:

$$
S_{e}^{p}=\frac{\sigma}{\mu} \alpha
$$

\section{Method to Predict Thermodynamic and Electronic Properties}

We therefore propose a method to quantitatively connect the theoretically and experimentally well-established electronic properties of molten semiconductor systems and the entropy of mixing. The following assumptions underlie the method, informed by the above discussions on the physics of semiconductors and thermodynamics of solutions: 
(1) The entropy of mixing is accounted for by the electronic entropy of mixing

(2) The Drude model of conductivity describes the conductivity of the system

(3) The Hall mobility is used for the carrier mobility

(4) The thermopower is dominated by the electronic contribution

(5) The total electronic entropy is approximated by $S_{e}^{p}$

Assumption (5) is read:

$$
S_{e}=S_{e}^{p}
$$

where $S_{e}$ is the total electronic entropyii ${ }^{\mathrm{ii}}$.

By means of example the method is tested to predict the thermopower of the tellurium (Te)-thallium ( $\mathrm{Tl})$ system at $800^{\circ} \mathrm{K}$. As one of the most studied molten semiconductor systems, both thermodynamic and electronic data are available for the tellurium-rich side of the phase diagram and for pure tellurium and thallium. The Hall mobility, thermopower, and electronic conductivity at $800^{\circ} \mathrm{K}$ are provided by Cutler as a function of composition [16-18].

The thermopower is connected to the total electronic entropy, according to Eqs. (9-11). The electronic entropy mechanical mixingiii term $S_{e}^{m}$ is obtained from the electronic entropy of the end-members:

$$
S_{e}^{m}=x_{T l} S_{e}^{T l}+x_{T e} S_{e}^{T e} .
$$

$S_{e}^{T l}$ and $S_{e}^{T e}$ are absolute electronic entropies obtained via Eqs. (6), (7), and (11) from the reported electronic properties of the end members in the molten state. We note that other methods are available to obtain the total electronic entropy of pure substances such as metals (i.e. 
ab initio atomistic modeling) [2]. The total electronic entropy is then obtained from the electronic entropy of mixing and mechanical mixing terms:

$$
S_{e}=\Delta S_{e}+S_{e}^{m}
$$

Substituting $S_{e}$ in Eq. (13) with Eqs. (9-11) and using the assumption that $\Delta S_{e} \approx \Delta S_{\text {mix }}$ (as discussed in the Appendix), the thermopower is obtained:

$$
\alpha=\frac{\mu}{\sigma}\left(\Delta S_{m i x}+x_{T e} n_{T e} e \alpha_{T e}+x_{T l} n_{T l} e \alpha_{T l}\right) .
$$

\section{Results for Tl-Te System}

We restrict our calculation to the tellurium-rich portion of the phase diagram, the only compositions for which Hall mobility measurements are available for the melt. The entropy of mixing $\left(\Delta S_{m i x}\right)$ is provided by Nakamura and Terpilowski from electromotive force (e.m.f.) measurements $[19,20]$. Figure 1 a shows the predicted thermopower of $\mathrm{Te}-\mathrm{Tl}$ from 0 to $67 \%$ atomic fraction of thallium (the congruent melting composition) by Eq. (14), compared to empirical data provided by Cutler.

Reversing the connection, we can demonstrate the utility of electronic property measurements of the molten state as a means to quantify entropy in the molten phase. We use reported empirical measurements of the mobility, electronic conductivity, and thermopower to calculate the entropy of mixing by Eq. (14). The results are shown in Fig. 1b against empirical data from Nakamura and Terpilowski and a model of the entropy of mixing developed by Oh based on Hillert's two-sublattice model and optimized with the data from Nakamura and Terpilowski. 
Figure $1 \mathrm{~b}$ shows that experimental uncertainty for e.m.f. measurements of the entropy of mixing for this system is on the order of $1 \mathrm{~J} \mathrm{~mol}^{-1} \mathrm{~K}^{-1}$. The modeled entropy of mixing by $\mathrm{Oh}$ is fit to this experimental dataset. The predicted entropy of mixing by Eq. (14) (the solid line in Fig. 1b) shows maximum deviation from the modeled data of less than $0.3 \mathrm{~J} \mathrm{~mol}^{-1} \mathrm{~K}^{-1}$, well within the range of experimental uncertainty of the e.m.f. method and without the use of any fitting procedure. Errors in the measurement of the electronic properties propagate through the model to errors in the predicted entropy of mixing. Specifically, a sensitivity analysis of error propagation for measurement of the thermopower shows that a $10 \%$ error in the thermopower propagates to roughly a $10 \%$ error in the entropy of mixing.

Measurements of thermopower in high temperature molten systems are typically reported with errors of less than $10 \%$ [21]. The conductivity is typically reported with measurement error of less than $1.5 \%$ [22]. Consequently, for systems such as Te-Tl, with entropies of mixing on the scale of $<5 \mathrm{~J} \mathrm{~mol}^{-1} \mathrm{~K}^{-1}$ that are primarily comprised of electronic entropy, the use of the present model using traditional experimental methods is expected to result in errors of $<0.5 \mathrm{~J} \mathrm{~mol}^{-1} \mathrm{~K}^{-1}$.

\section{Discussion on the Validity of the Model}

We made the assumption that the total electronic entropy is approximated by $S_{e}^{p}$ (Eq. (11)). This, as shown by Rockwood for the case of a free electron system, is not always an appropriate assumption [7]. However, as shown in Figures $1 \mathrm{a}$ and $1 \mathrm{~b}$, our assumption led to the accurate prediction of macroscopic properties of a molten semiconductor system. We interpret the results to indicate the validity of assumption (5) for molten semiconductor systems.

Further, as shown in Figures 1a and 1b, the predicted total electronic entropy of mixing approximates the total entropy of mixing for the Te-Tl system. This validates assumption (1), in 
accordance with our expectations based on macroscopic thermodynamic considerations (Appendix): the electronic entropy is critical to the thermodynamics of mixing for molten semiconductors.

The total electronic entropy is related to the accessible density of states (DOS) of electrons; only states vicinal to the chemical potential of electrons (or the Fermi level) are accessible and substantially contribute to the electronic entropy of the system.

Measuring the thermopower of a system therefore consists of measuring the response of the electronic component of the entropy to a perturbation in temperature. This measurement is not without analogy to electromotive force (e.m.f.) measurement, one of the few experimental methods to determine the (total) entropy of a system by monitoring a relative chemical potential difference as a function of temperature [23].

If the thermopower of a system is quantifiably related to the electronic entropy and gives access to the absolute electronic entropy of a system then the thermopower can be considered a material property with physical meaning for a material at equilibrium $[23,24]$.

If Eq. (6) is valid for semiconductor systems, then we may draw an interesting analogy between the entropy of transport used in the irreversible thermodynamics derivation of the thermopower and the electronic entropy, a reversible thermodynamic quantity. In irreversible thermodynamics, the thermopower reflects the entropy transported during the thermally driven motion of charged particles. The thermopower is then defined as the entropy per unit charge associated with mobile electrons in the system [25]. 
Therefore, the thermopower of a material and the number of charged particles that contribute to nonlocal transport phenomena (i.e. mobile electron density $n$ of charge $e$ ) provide a quantification of the total transported entropy of a system $\left(S_{t}\right)$ :

$$
S_{t}=-n e \alpha
$$

in units of J m${ }^{-3} \mathrm{~K}^{-1}$ which is identical to Eq. (6). From the equivalence, we can conclude that, for molten semiconductor systems, the irreversibly defined transport entropy is equal to the reversibly defined total electronic entropy:

$$
S_{t}=S_{e} .
$$

Rockwood discusses the implications of the connection between irreversible and reversible thermodynamic quantities in [10].

\section{Extension to Solids and Other Semiconductor Systems}

It is foreseen that the connection of the electronic properties to electronic entropy will provide novel insights even if some of the assumptions of the method do not hold, such as the total entropy of mixing being approximated by the electronic entropy of mixing, the Drude model approximating the conductivity of the system, or the validity of Eq. (11). To illustrate the value of the connection, it is applied to predict the entropy of fusion, and in particular the electronic entropy of fusion $\Delta S_{f}$ of solid semiconductor compounds - a property for which there exist more data at present. To apply the proposed model, we use available data on the electronic properties of the solid and molten compound at melting and apply Eqs. (9) and (10). We focus on binary systems for which these data, as well as electronic entropy of fusion data, are available in the literature. 
Figure 2 plots the predicted electronic entropy of fusion via Eqs. (9) and (10) vs. the electronic entropy of fusion as provided by Belotskii [26].

Figure 2 shows that for certain systems Eqs. (9) and (10) provide a reasonable prediction of the reported value of the electronic entropy of fusion (solid symbols). However, the prediction can overestimate and underestimate the reported value of the entropy of fusion (hollow circles). Addressing these errors in turn helps to illustrate the value of the correlation.

The two compounds for which Eqs. (9) and (10) substantially overestimate the electronic entropy of fusion are AlSb and GaSb. Hall measurements of AlSb and GaSb report electron densities of $7.0 \times 10^{21} \mathrm{~cm}^{-3}$ and $4.1 \times 10^{22} \mathrm{~cm}^{-3}$ respectively, as opposed to $2.0 \times 10^{23} \mathrm{~cm}^{-3}$ and $2.2 \mathrm{x}$ $10^{23} \mathrm{~cm}^{-3}$ predicted by the Drude model of conductivity (Eq. (8)) [27]. Adopting the measured electron density of GaSb and applying Eqs. (6) and (7) predicts an electronic entropy of fusion of $14.5 \mathrm{~J} \mathrm{~mol}^{-1} \mathrm{~K}^{-1}$ vs. the literature provided value of $16.7 \mathrm{~J} \mathrm{~mol}^{-1} \mathrm{~K}^{-1}-$ a prediction within the anticipated error of the reported values [26]. The corrected value is shown in Fig. 2 (solid blue symbol). Not only does this demonstrate the validity of the proposed connection for systems whereby the Drude model is inapplicable, it demonstrates the utility of the connection to determine which electronic model is best suited for systems where thermodynamic data are available. This is particularly valuable since Hall measurements for molten systems are not as common as for their solid counterparts. We have compiled these data and provided corrected predictions via Eqs. (6) and (7) in Fig. 2 as available (blue symbols).

The model does not accurately account for the entirety of the electronic entropy of fusion for some compounds. We put forth three explanations for this. The first, described above, is the lack of an accurate model for the conductivity of those systems in the molten state. As shown in 
Fig. 2, where a correction to the Drude model is applied, a systemic under-prediction of electronic entropy remains. A second explanation is the lack of validity of our assumption reflected in Eq. (11) (i.e. there are contributions to the total electronic entropy that are not accounted for by $S_{e}^{p}$ ). A third explanation for the remaining discrepancy lies in the existence of a second contribution to the electronic entropy, not accounted for by the connection put forth. As indicated in Section 2, the electronic entropy described in the correlation stems from the size of the available state space of the electrons near the Fermi level. It does not take into account the configurational electronic entropy, since it does not manifest in the thermoelectric power. Configurational electronic entropy describes the configurational entropy of localized electrons and holes.

In analogy to the configurational entropy of a solid solution on a lattice, the configurational electronic entropy is described by Zhou et. al. [28]. The configurational electronic entropy has been shown to substantially contribute to the electronic entropy and the total entropy of mixing for certain classes of systems, such as mixed valence oxides [20] or certain semiconductor systems at melting [29]. Germanium is for example modeled to have a configurational electronic entropy of fusion of approximately $22.1 \mathrm{~J} \mathrm{~mol}^{-1} \mathrm{~K}^{-1}$, an amount that bridges the electronic entropy of fusion predicted by our model and the reported value. The combined predicted electronic entropy of fusion is shown in Fig. 2 (red symbol).

Whereas the configurational contribution to total electronic entropy stems from electrons that are localized, the electronic entropy described by Eqs. (6) and (7) describes the entropy of delocalized electrons. We shall henceforth call this quantity the electronic state entropy. This terminology arises from the number of available states, or density of states (DOS), of a system near the Fermi level (as accessible according to the Fermi-Dirac distribution). Thus, the 
configurational electronic entropy reflects statistics of distinguishable particles whereas the electronic state entropy reflects the statistics of indistinguishable particles. For systems such as alkali metals, where the DOS is often small near the Fermi level, or for ionic systems, where the DOS is often zero near the Fermi level, the contribution of the electronic state entropy to the total electronic entropy is small. However, for systems such as semiconductors (or the subclass of thermoelectrics that are designed to have maximal density of states near the Fermi level), there can be a substantial contribution of the electronic state entropy due to the large DOS near the Fermi level. Thus, it is for molten semiconductors that Eq. (14) produces the best approximation for the total entropy of mixing.

Hence, the provided model reflects the electronic state entropy as it manifests in the DOS near the Fermi level, and to date does not include a configurational component arising from localization of electrons or holes.

\section{Extension to Metallic Systems}

The DOS directly reflects the chemical ordering (bonding) of a system [30]. Consequently, it would be surprising if an empirically accessible quantity that reflects the DOS near the Fermi level (i.e. the thermopower) did not give at least qualitative access to the ordering tendencies of a system. We have demonstrated above that, in the case of molten semiconductors, the electronic entropy dominates the entropy of mixing and hence substantially reflects the ordering of a system. However, even for cases where the electronic entropy of mixing is not a dominant component of the total entropy of mixing, the electronic entropy will still reflect an evolution in the chemical ordering of a system. 
Figure 3 shows the predicted electronic contribution to the entropy of mixing (black circles) in the molten state according to Eq. (14) of the Mg-Bi system at $1100^{\circ} \mathrm{K}$ and the empirically validated total entropy of mixing (black line) [31]. The electronic property data is provided by Ratti and Enderby [13,32]. The Mg-Bi system is metallic across the entire range of composition in the molten phase.

The total entropy of mixing for this system is on the scale of $6 \mathrm{~J} \mathrm{~mol}^{-1} \mathrm{~K}^{-1}$, confirming that the electronic entropy does not describe the entire thermodynamics of mixing of the $\mathrm{Mg}-\mathrm{Bi}$ system. However, the electronic state entropy of mixing suggests ordering at a composition of $40 \% \mathrm{Bi}$. The phase diagram of the Mg-Bi system indeed exhibits a congruent melting compound at $\mathrm{Mg}_{3} \mathrm{Bi}_{2}$ [33].

Thus, the electronic entropy, as analyzed through the proposed correlation and empirically probed by measurement of the electronic properties of the melt, also reflects the chemical ordering of metallic systems. When measured near the liquidus, these properties are a proxy for ordering of the corresponding solid phases. It is therefore put forth that measurement of electronic properties of melts can provide fast and simple access to high-temperature solid phase ordering.

\section{Extension to Ionic Systems}

The above connection between the measured thermopower and the electronic entropy of a system assumes that the electronic contribution to the thermopower dominates. However, in some systems (especially ionic systems), the Soret effect substantially contributes to the measured thermopower. The Soret effect reflects the chemical response of the system to a perturbation of temperature. For such a situation, Carl Wagner has provided a comprehensive method and 
analysis to isolate the electronic and ionic contribution to the thermopower [23]. We note that as electronic thermopower reflects a change in the contribution of (delocalized) electron entropy to the free energy as a function of temperature, the Soret effect reflects the contribution of chemical species entropy to the free energy as a function of temperature. We propose that, by analogy, the partial entropy of species of systems exhibiting the Soret effect should be accessible via measurement of the thermopower. Rockwood and Tykodi discuss further implications of the ability to measure electronic entropy on the ability to quantify reversible thermodynamic quantities of ionic melts that were previously inaccessible $[7,11]$.

\section{Conclusion}

We have proposed a quantitative connection between thermodynamic properties (entropy) and electronic properties (thermopower) of systems. The model quantitatively predicts the entropy of mixing of molten semiconductor systems. Electronic entropy is shown to reflect ordering in molten systems and the solid phase, even for systems in which the electronic entropy of mixing does not substantially contribute to the total entropy of mixing. The model has been shown to have consequences in the interpretation of the connection between quantities from irreversible and reversible thermodynamics. It is proposed that an analogous method using the Soret effect can quantify partial entropies of chemical species in high temperature systems. 
Table 1 summarizes the importance of electronic entropy for different classes of systems, and the materials information that can be derived from its experimental measurement by the method put forth in this work. 


\section{Acknowledgements}

This work was supported by the AFOSR under Grant FA9550-15-1-0046. We would like to acknowledge the role of the Fannie and John Hertz Foundation for support of the researchers and Dr. Ali Sayir and the AFOSR for their financial support. 


\section{Endnotes}

${ }^{i}$ For systems that exhibit ambipolar conductivity a similar equation with contributions from both electrons and holes is used. We simplify for purpose of demonstration to systems that exhibit monopolar conductivity.

ii For a discussion on the validity of decomposing the free energy function into components see Smith [34]

iii Mechanical mixing entropy is the concentration-weighted entropies of the end-members of a system in absence of any chemical interactions. Thermodynamic mixing functions are referenced to mechanical mixing: $\Delta X_{m i x}=X-X^{m}$.

iv See Wagner for a discussion on isolating the electronic contribution to thermopower [23] 


\section{References}

[1] B. Fultz, Prog. Mater. Sci. 55, 247 (2010).

[2] B. Fultz, Phase Transitions in Materials (Cambridge University Press, 2014).

[3] M. R. Peterson and B. S. Shastry, arXiv (2010).

[4] P. M. Chaikin and G. Beni, Phys. Rev. 13, (1976).

[5] D. C. Wallace, Statistical Physics of Crystals and Liquids (World Scientific, 2002).

[6] A. Rockwood, Phys. Rev. A 30, 2843 (1984).

[7] A. Rockwood, Electrochim. Acta 112, 706 (2013).

[8] A. Rockwood, J. Mod. Phys. 199 (2016).

[9] R. B. Roberts, Philos. Mag. 36, 91 (1977).

[10] A. Rockwood, Electrochim. Acta 107, 686 (2013).

[11] R. Tykodi, Thermodynamics of Systems in Nonequilibrium States (Thinkers' Press, 2002).

[12] S. Kjelstrup, Electrochem. Acta 107, 693 (2013).

[13] J. E. Enderby and A. C. Barnes, Rep. Prog. Phys. 53, 85 (1990).

[14] A. F. Ioffe and A. R. Regel, Progress in Semiconductors, v. 4 (1960).

[15] N. F. Mott and E. A. Davis, Electronic Processes in Non-Crystalline Materials (Clarendon Press, 1971).

[16] J. Donally and M. Cutler, Phys. Rev. 176, 1003 (1968).

[17] M. Cutler, Liquid Semiconductors (Academic Press, 1977).

[18] M. Cutler and C. E. Mallon, J. Chem. Phys. 37, 2677 (1962). 
[19] C. Oh, J. Phase Equilibria 14, 197 (1993).

[20] Y. Nakamura and M. Shimoji, Trans. Faraday Soc. 67, 1270 (1971).

[21] S. Ohno, A. C. Barnes, and J. E. Enderby, J. Phys. Condens. Matter 6, 5335 (1994).

[22] V. P. Didoukh, Y. O. Plevachuk, and B. I. Sokolovskii, Int. J. Thermophys. 20, 343 (1999).

[23] C. Wagner, Prog. Solid State Chem. 7, 1 (1972).

[24] D. Adler, Physics of Disordered Materials (Plenum Press, New York, 1985).

[25] H. B. Callen, 333, (1948).

[26] D. P. Belotskii and O. N. Manik, J. Thermoelectr. 1, 32 (2004).

[27] A. R. Regel, V. M. Glazov, and A. A. Aivazov, Sov. Phys. Semicond. 8, 1398 (1975).

[28] F. Zhou, T. Maxisch, and G. Ceder, Phys. Rev. Lett. 97, 155704 (2006).

[29] B. K. Chakraverty, Radiat. Eff. 4, 39 (1970).

[30] C. Kittel, Introduction to Solid State Physics, 7th ed. (Wiley, 1996).

[31] R. Hultgren, R. Orr, P. Anderson, and K. Kelley, Selected Values of Thermodynamic Properties of Metals and Alloys (Wiley, 1963).

[32] V. Ratti and A. B. Bhatia, J. Phys. F 5, 893 (1975).

[33] A. Nayeb-Hashemi and J. B. Clark, Bull. Alloy Phase Diagrams 6, 528 (1985).

[34] P. E. Smith and W. F. Van Gunsteren, J. Phys. Chem. 13735 (1994).

[35] V. M. Glazov, Liquid Semiconductors (Plenum Press, 1969). 


\section{Appendix}

For simplicity, a binary solution of $\mathrm{A}$ and $\mathrm{B}$ that defines a molten semiconductor system is considered, though the formalism is not restricted to binary systems. For a solution in the A-B system, the Gibbs free energy of mixing $\left(\Delta G_{m i x}\right)$ is the difference between the Gibbs free energy $(G)$ of the actual solution and the mechanical Gibbs free energy $\left(G^{m}\right)$ of the end-members A and B, defined as:

$$
G^{m}=x_{A} G_{A}+x_{B} G_{B} .
$$

$\Delta G_{m i x}$ is conventionally expressed in terms of the enthalpy and entropy of mixing, respectively $\Delta H_{\text {mix }}$ and $\Delta S_{\text {mix }}$, as:

$$
\Delta G_{\text {mix }}=G-G^{m}=\Delta H_{\text {mix }}-T \Delta S_{\text {mix }} .
$$

The entropy of mixing can be delineated into contributions from configurational $\left(\Delta S_{c}\right)$, vibrational $\left(\Delta S_{v}\right)$, electronic $\left(\Delta S_{e}\right)$, and other terms $\left(\Delta S_{r}\right)$ :

$$
\Delta S_{m i x}=\Delta S_{c}+\Delta S_{v}+\Delta S_{e}+\Delta S_{r} .
$$

$\Delta S_{e}$ is typically of substantial magnitude for molten semiconductor systems (see Table A1). The configurational entropy of mixing $\left(\Delta S_{c}\right)$ is determined by the degree of chemical ordering present in a system. The less ordered a system, the larger the configurational entropy of mixing. Molten semiconductors, as presented above, are ordered systems and consequently have minimal configurational entropy of mixing. Such a statement can be substantiated by consideration of the

phase diagram. Molten semiconductor systems typically exhibit a compound melting congruently and liquid-phase miscibility gaps. Directly above the congruent melting point the system exhibits 
short-range order nearly identical to the solid compound $(\mathrm{AB})$. The atoms of $A$ and atoms of $B$ are fully associated with each other at the $\mathrm{AB}$ composition, thus minimizing configurational entropy of mixing. Between the congruent melting point and the end members are typically miscibility gaps. For compositions within these miscibility gaps the system segregates into two non-mixing phases, which further reduces the configurational entropy of mixing. Consequently, $\Delta S_{c}$ is typically of minor magnitude and the result is that configurational entropy plays a small role in the entropy of mixing for molten semiconductor systems:

$$
\left|\Delta S_{c}\right| \ll\left|\Delta S_{e}\right| .
$$

The lack of long-range order in molten systems leads to a drastic reduction of the magnitude of the vibrational contribution to the entropy in comparison with solids [1]. $\Delta S_{v}$ for molten systems reflects the difference between vibrational entropy of the molten system and the mechanical mixture of the two end members (referenced to the liquid state) - the absolute magnitude of which is not significant for the majority of molten semiconductor systems:

$$
\left|\Delta S_{v}\right| \ll\left|\Delta S_{e}\right| .
$$

$\Delta S_{r}$ may comprise magnetic entropy and other system-specific entropic effects, which are ignored at the present stage.

TABLE A1. Typical entropies of mixing for molten semiconductor systems [26,27,35]

\begin{tabular}{ll}
\hline \hline & Value for Molten \\
Semiconductors
\end{tabular}

$\Delta \boldsymbol{S}_{e}\left(\mathrm{~J} \mathrm{~mol}^{-1} \mathrm{~K}^{-1}\right) \quad \sim 3$




\begin{tabular}{ll}
$\Delta \boldsymbol{S}_{c}\left(\mathrm{~J} \mathrm{~mol}^{-1} \mathrm{~K}^{-1}\right)$ & $<1$ \\
$\Delta \boldsymbol{S}_{v}\left(\mathrm{~J} \mathrm{~mol}^{-1} \mathrm{~K}^{-1}\right)$ & $<1$ \\
$\Delta \boldsymbol{S}_{r}\left(\mathrm{~J} \mathrm{~mol}^{-1} \mathrm{~K}^{-1}\right)$ & $<1$ \\
\hline
\end{tabular}




\section{Tables}

Table 1. Role of electronic entropy for various molten material systems

\begin{tabular}{lccc}
\hline & Metallic & & Ionic \\
& & Semiconducting & \\
\hline Reflects ordering & Yes & Yes & Yes \\
Approximates & No & Yes & No \\
$\left(\Delta \boldsymbol{S}_{\text {mix }}\right)$ & & & \\
$\begin{array}{l}\text { Empirically } \\
\text { accessible in melt }\end{array}$ & Yes & Yes & Yesiv \\
\hline \hline
\end{tabular}




\section{Figure Captions}

Figure 1. (a) thermopower vs. atomic fraction thallium at $800^{\circ} \mathrm{K}$ as predicted by Eq. (14) (solid

line) and as measured by Cutler (blue circles) [17]. (b) entropy of mixing vs. atomic fraction thallium at $800^{\circ} \mathrm{K}$ as predicted by Eq. (14) (solid line), as measured by Terpilowski (red circles), as measured by Nakamura (blue circles), and as calculated by Oh using the two-sublattice Hillert model optimized with data from Terpilowski and Nakamura (dashed line) $[19,20]$

Figure 2. The predicted vs. reported electronic entropy of fusion [26]. Solid symbols show predictions that agree with reported values. Hollow symbols show predictions that substantially deviate from reported values. Black symbols show predictions using Eqs. (9) and (10). Blue symbols show predictions using Eqs. (6) and (7) and measured values for the number of carriers. The red symbol shows the predicted entropy of fusion for Ge including the configurational electronic entropy of fusion.

Figure 3. The predicted electronic entropy of mixing of the Mg-Bi system at $1100^{\circ} \mathrm{K}$ by Eq. (14) (black circles) and literature reported values of the total entropy of mixing (solid line) [31] 
Figure 1
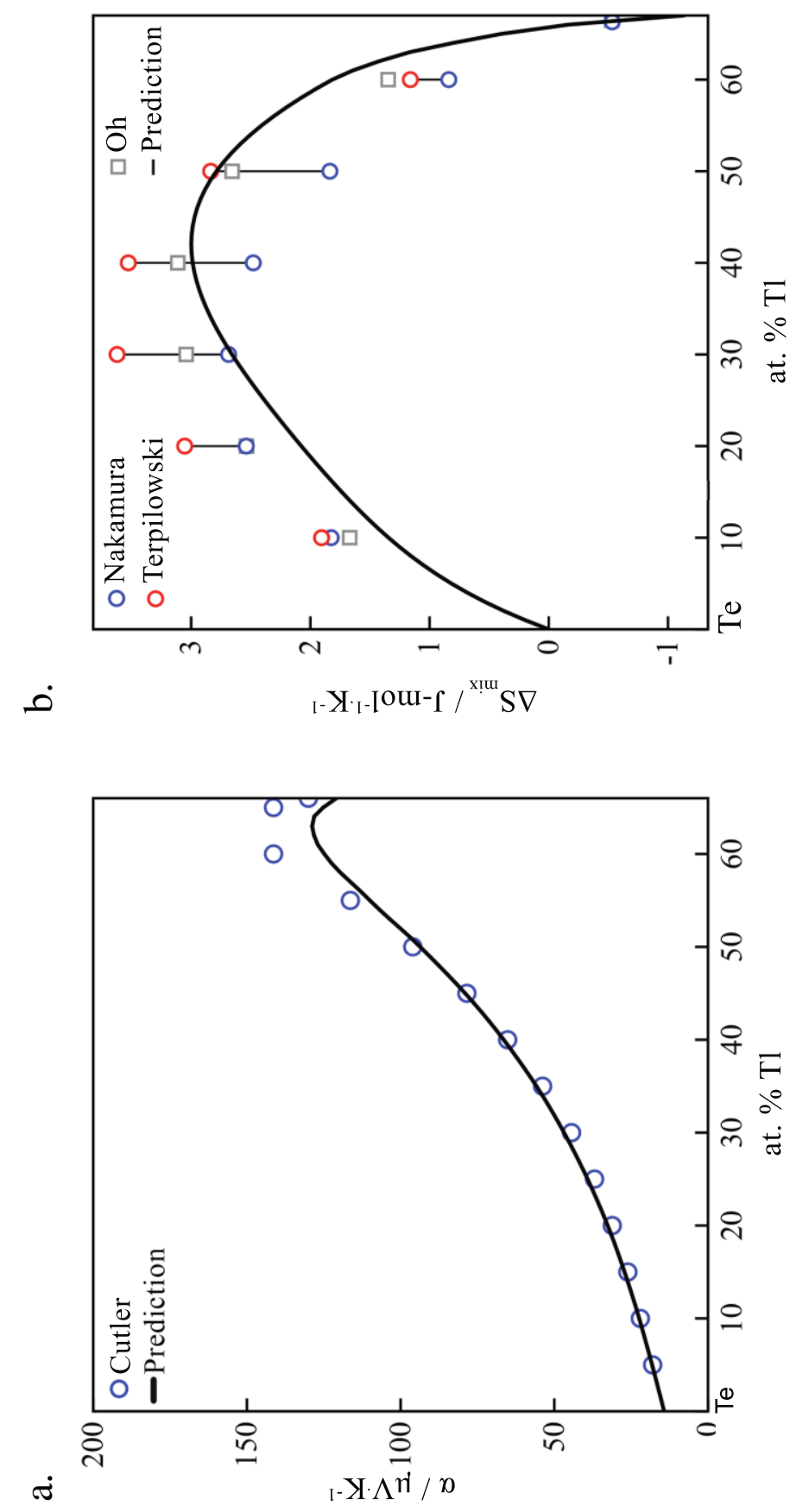
Figure 2

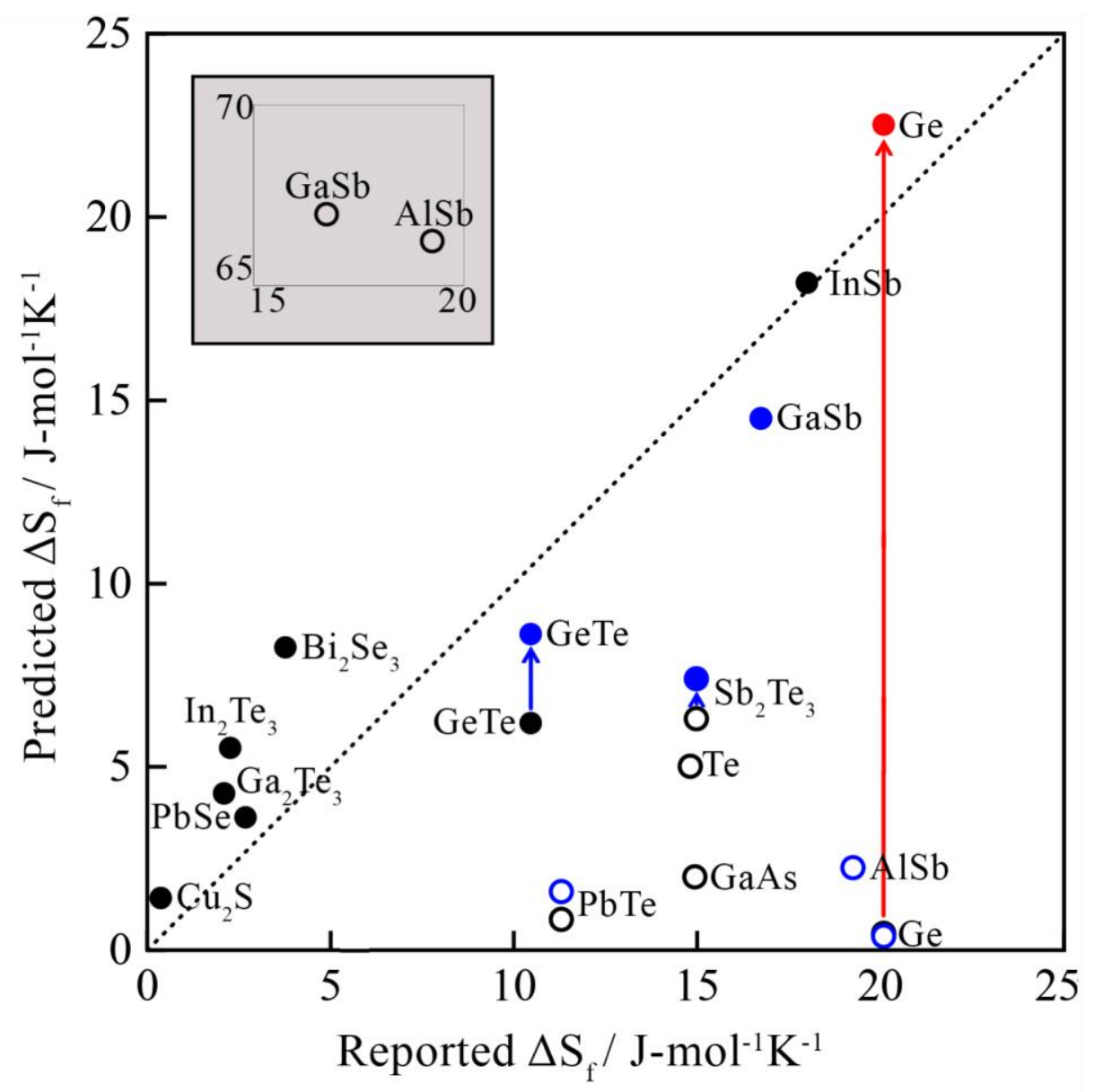


Figure 3 


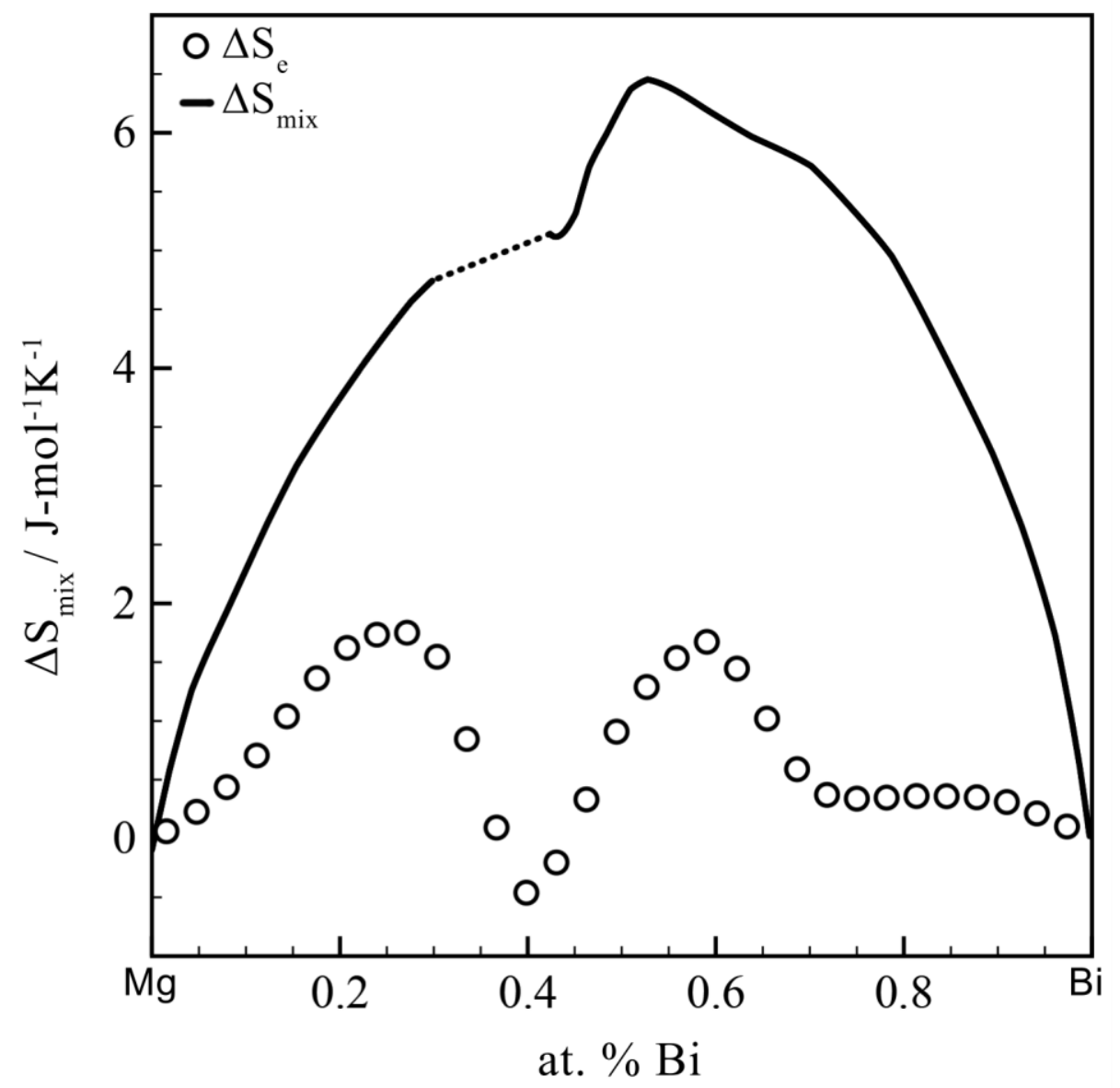


